\title{
An overview of benefit/risk of disease modifying treatment of rheumatoid arthritis as of today*
}

\author{
HAROLD E PAULUS \\ From the Department of Medicine, UCLA School of Medicine, Los Angeles, California 90024, USA
}

SUMmARY In chronic rheumatoid arthritis (RA), disease modifying drugs are used in an attempt to suppress the progressive damage to tissues and joints that is associated with active disease. Their success in achieving this goal is variable; responses vary from complete suppression of all signs and symptoms of RA to continued active disease, with progressive disability, despite prolonged therapy. Because disease activity almost always recurs after the therapy is stopped, early interruption of an effective therapy for any reason will make its benefit insignificant in a lifelong disease such as RA. Similarly, short-term sequential use of multiple disease modifying therapies is unlikely to be beneficial. The immediate problems with these therapies are substantial. In general, fewer than $50 \%$ of patients are able to continue a particular drug for more than one year. Since it takes three to 12 months or longer to achieve maximum effects, those patients who are unable to continue the drug receive little benefit from it. Inevitable delayed side-effects, such as those associated with chronic corticosteroid therapy, may make the benefit/risk ratio unacceptable. Potential late lethal adverse effects, such as malignancy, weight the benefit/risk ratio to varying extents for individual patients, depending on the relative probability that the adverse effect will occur during the remainder of the patient's anticipated life span, and are of greater importance in younger patients. In that minority of patients who achieve remission or near remission and are able to tolerate a disease modifying treatment for many years, it is of truly significant benefit. We are still searching for a therapy that will reliably achieve this goal for most patients with RA.

The purpose of disease modifying antirheumatic drug (DMARD) therapy of rheumatoid arthritis (RA) is to:

(1) reduce, suppress, or eliminate disease activity now; and

(2) thereby prevent the extended and perhaps permanent effects of disease activity. ${ }^{42}$

"Supported in part by USPHS Grant NO1-AM6-2218.
Currently available DMARDs include organic gold compounds, antimalarial drugs (chloroquine and hydroxychloroquine), D-penicillamine and the immunosuppressants (azathioprine, 6-mercaptopurine, cyclophosphamide, chlorambucil and methotrexate). Investigational disease modifying therapies include the $\mathrm{T}$ cell stimulants levamisole and thymopoietin, lymphocyte depletion or suppression by thoracic duct drainage, lymphopheresis, antithymocyte globulin, or total lymphoid irradiation and removal of plasma constituents by plasmapheresis.

These interventions are remarkably diverse in their chemical and pharmacological properties ando응 effects. Yet, they appear to be remarkably similar in their effects in patients with RA. Their administration does not result in any observable immediate amelioration of the signs or symptoms of RA, such as one expects to see with corticosteroids or nonsteroidal anti-inflammatory drugs. However, after weeks or months of treatment, a subtle and somewhat questionable decrease in the severity of symptoms may occur. With continued therapy, the average patient demonstrates overall moderate suppression of disease activity, but this average includes some patients who have no improvement at all, and others who eventually achieve complete, or nearly complete, remission of disease manifestations, often accompanied by normalisation of laboratory abnormalities that are associated with disease activity, and stabilisation of $x$-ray evidence of joint damage. However, these beneficial effects are not permanent. When the disease modifying treatment is stopped benefit usually persists for weeks or months, but then disease manifestations gradually recur in the same delayed and subtle fashion as they had disappeared when the therapy was started.

How well do we succeed in our goal of preventing the progressive damage to tissues and joints that is associated with disease activity using the DMARDs that are available today? What price do our patients pay for these benefits?

Chronic RA is usually a life-long disease. If we expect to restore the patient to normal, productive 
life with a DMARD induced remission, that remission must be sustained for many years. Since it generally takes three to 12 months or longer to achieve maximum benefit with DMARD therapy, patients who are unable to take the drug for more than one year receive little benefit from it.

\section{Benefits}

In evaluating the long term benefits of DMARDs on the life quality of patients with RA, the classic published placebo-controlled double-blind studies that have established the use of these drugs are of little value because, in order to avoid unnecessarily prolonged administration of placebo, they are generally of the shortest possible duration, usually no more than four to 12 months. $^{346371140155224}$ However, there are some published reports on the long-term usefulness of some of the DMARDs.

\section{GOLD COMPOUNDS}

Srinivasan $e t a^{205}$ reported our findings in a cohort study of 111 patients who were followed for an average of three years after starting gold therapy. Fiftyeight $(52 \%)$ patients stopped gold within the first 18 months. Of those who continued gold for more than 18 months, $83 \%$ achieved remission, although five of these subsequently flared while continuing gold therapy. Thus, of the 111 patients starting gold, about $35 \%$ had major sustained improvement an average of three years later.

Rothermich and associates ${ }^{189}$ started 97 patients in a prospective study of gold therapy. After an average of $1 \frac{1}{2}$ years, $41 \%$ were having sufficient clinical benefit to continue the drug. However, four to six years after the study was started, only 14 patients were continuing gold therapy, and two more had discontinued because of remission. Most stopped gold because of loss of benefit, or toxicity, or both simultaneously.

In the same report, Rothermich et al ${ }^{189}$ examined 171 patients who started D-penicillamine after failing gold therapy and $53 \%$ had stopped gold within the first year, more for toxicity than for lack of benefit. At the end of three years, only $20 \%$ continued gold, somewhat fewer than Srinivasan's finding of $35 \% .{ }^{205}$ At the end of five years, only $9 \%$ were still taking gold. Of course, these figures are biased by the requirement that only patients who had stopped gold were counted; the analysis is completely retrospective and the population may not be representative. Nevertheless, the majority of these patients stopped gold for loss of benefit.

\section{D-PENICIL LAMINE}

Several similar studies of D-penicillamine therapy have been reported recently. Kean et al ${ }^{112}$ found that $47 \%$ of 101 patients continued D-penicillamine after one year and $38 \%$ after two years. The major reason for stopping therapy in the first year was toxicity. After two years, two patients were in remission, five in partial remission, and 31 had $75 \%$ improvement.

Webley and Coomes ${ }^{233}$ reported that $46 \%$ of 114 patients continued penicillamine after an average of $10 \cdot 1$ months' treatment. Of the 62 withdrawals, 40 were for toxicity and 12 for lack of response. There were no differences in toxicity or improvement rates in patients receiving more or less than $600 \mathrm{mg}$ daily, or in patients who had or had not had previous gold therapy.

\section{COMPARATIVE STUDIES}

Husain and Runge ${ }^{96}$ evaluated the risks of termination of treatment with gold, penicillamine, hydroxychloroquine and levamisole using a life table analysis. The risks for termination of gold and penicillamine were similar to those reported above, while that for hydroxychloroquine ( $25 \%$ in 12 months) was lower, and that for levamisole (57\% in 12 months) higher than for the other two drugs. However, in another paper, the same group reported that the median termination time was 60 months for gold and 13 months for antimalarial compounds. Lack of efficacy was responsible for treatment termination in $41 \%$ of the antimalarial group in this study in which treatment was frequently terminated for lack of response after three to six months of treatment. ${ }^{185}$

Dwosh et $a l^{56}$ randomly assigned patients with class II RA to azathioprine, gold or chloroquine. After six months of treatment, all three agents were comparable in terms of toxicity and clinical improvement. However, the chloroquine group did not maintain continued improvement beyond six months, while in the gold and azathioprine groups benefit continued for up to 44 months.

Hoh et $a l^{93}$ reported the incidence of remissions of RA in patients treated with gold, penicillamine, or chloroquine for at least one year. Prolonged remissions occurred in only $10 \%$ of the 230 drug courses, while $59 \%$ had modest partial responses and the remainder had no response.

Currey et $a l^{38}$ compared azathioprine, cyclophosphamide and gold in a well-designed 18-month double-blind randomised trial of 121 patients. They concluded that the two immunosuppressives produced clinical improvement comparable to that with gold. Cyclophosphamide was perhaps marginally more effective. Drug management was easiest with azathioprine. However, only $25 \%$ of patients on azathioprine, $36 \%$ on cyclophosphamide and $24 \%$ on gold completed 18 months of treatment. Of course, withdrawals for toxicity were increased by the 
double-blind nature of the trial and the inclusion of gold, which necessitated withdrawing seven azathioprine and 11 cyclophosphamide patients for marrow depression.

On the other hand, Cade et $^{2}{ }^{25}$ reported that 14 of 16 patients treated with azathioprine for two to five years achieved $100 \%$ work ability, although it took up to 32 months of treatment to achieve that maximum response. Furthermore, serial $x$-rays showed no progression of destruction after completion of the second year of therapy, even when followed for as long as eight years.

\section{Risks}

Only major risks will be evaluated here. It is obvious that patients who are forced to stop treatment because of temporary reversible rash, proteinuria, marrow suppression, drug induced hepatitis, etc, do not benefit from the drug, but these adverse reactions rarely shorten their life span or produce permanent damage.

\section{E A T H}

Davis $^{40}$ has recently reviewed the undesirable effects of gold therapy. With current dosages, marrow aplasia is the most serious adverse effect. Over a seven-year period in Great Britain, 16 deaths related to gold therapy were found, an estimated incidence of 1.6 deaths per 10000 prescriptions. This figure suggests to Davis that gold is ten times more toxic than any other therapy used in Great Britain. Davis reviewed four studies, published in 1962, 1968, 1976 , and 1977 , reporting a total of 50 patients with gold induced marrow aplasia, which was fatal in $66 \%$.

Similarly, Kay ${ }^{111}$ collected information on ten patients in Great Britain with pancytopenia associated with penicillamine therapy, six of whom died. The dose range was 250 to $1000 \mathrm{mg} /$ day with an average of $615 \mathrm{mg}$. The duration of therapy was from three to 60 months with a mean of 16 months. She reports that the Committee of Safety of Medicines had had reported to them 18 deaths from D-penicillamine from 1964 to 1977 (three of whom were included in the six deaths reported by Kay). Fourteen of the 18 deaths were due to blood dyscrasias.

Similarly, deaths have occurred in patients who have developed agranulocytosis while taking levamisole. ${ }^{147}$

Lewis et al ${ }^{125}$ reported on the causes of death in 311 patients with RA who were observed over an 11-year period. A total of 46 patients died, 13 of them from malignancies. The incidence of deaths from neoplasia was not greater in 214 patients treated with azathioprine and/or chlorambucil or cyclophosphamide
$(2.8 \%)$ than in 97 patients who did not receive a cytotoxic drug $(7 \%)$.

Isomaki et $a l^{102}{ }^{101}$ reported two large studies of causes of death in patients with RA in Finland. In the first, an age and sex matched comparison of 1000 patients with RA and 1000 control subjects, malignant neoplasms were less frequent in RA (9\% of deaths; $1.1 \%$ of the population) than in the controls ( $30 \%$ of deaths; $2.1 \%$ of the population). In the second study of more than 45000 patients with RA, the risk of death from lymphoma, leukaemia or myeloma was twice as great in RA (130 deaths) as that expected in the general population (59 deaths). No data are given regarding the drugs used by these patients.

O THE R S ERIOUS TOX I C ITIE S

Maculopathy occurred in $10 \%$ of 222 patients treated with $800 \mathrm{mg} /$ day hydroxychloroquine or 500 $\mathrm{mg} /$ day chloroquine by Dubois for systemic lupus erythematosus, but after 10 years of follow-up, visual acuity was adequate in all but two of the patients. ${ }^{21}$ However, with $400 \mathrm{mg}$ daily for more than one year in 99 patients, no loss of vision occurred. ${ }^{190}$

Sterility occurs with cyclophosphamide and chlorambucil, haemorrhagic cystitis with cyclophos- phamide and hepatic fibrosis with methotrexate. All of the immunosuppressive drugs are thought too increase susceptibility to infection, particularly when combined with large doses of corticosteroids.

Induction of autoimmune diseases has been reported with penicillamine therapy for RA, and also for Wilson's disease. In addition, the renal lesions associated with gold induced proteinuria have the characteristics of an immune complex mediated nephropathy, and the agranulocytosis of levamisole appears to be immune mediated. We have also seen new autoimmune manifestations develop during the apparently successful treatment of RA with thoracic duct lymphocyte depletion. These observations suggest that, while correcting some disease related abnormalities of the immune system, we may perturb the system in such a way that other manifestations of autoimmune disease emerge in some patients.

\section{Conclusions}

For those patients who achieve remission with a DMARD and are able to tolerate it for many years, the benefit clearly is greater than the risk. Unfortunately, because most patients do not achieve optimal benefits, or must stop treatment for annoying sideeffects, our goal of favourably altering the course of their RA is not achieved. Except for the antimalarials, there appears to be a real, although small, risk of drug associated death with all of the available 
DMARDs. However, the available data are not suffi-. cient to produce a satisfactory rank order of this risk with the various drugs.

We have no reason to be satisfied with our present accomplishments with DMARD therapy. However, it seems clear that DMARDs affect either the cells that participate in immune responses or their products. With increasing knowledge about this system and its methods of communication, and increasing attention to the antigens associated with autoimmune diseases, perhaps future DMARDs will be more effective. 